\title{
Interobserver variability of ultrasound parameters in portal hypertension
}

\author{
Patricia Moreno Sebastianes ${ }^{1}$, Danilo Moulin Sales ${ }^{1}$, José Eduardo Mourão Santos ${ }^{1}$,

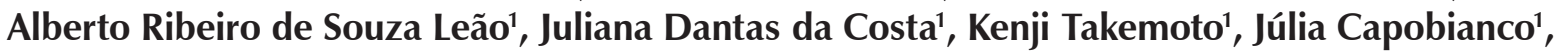 \\ Alexandre Sérgio de Araújo Bezerra² ${ }^{2}$ Giuseppe D'Ippolito ${ }^{1 /+}$
}

${ }^{1}$ Departamento de Diagnóstico por Imagens, Universidade Federal de São Paulo, Rua Napoleão de Barros 800, 04024-002 São Paulo, SP, Brasil ²Faculdade de Medicina, Universidade de Brasília, Brasília, DF, Brasil

The aim of this study was to assess interobserver agreement of ultrasound parameters for portal hypertension in hepatosplenic mansonic schistosomiasis. Spleen size, diameter of the portal, splenic and superior mesenteric veins and presence of thrombosis and cavernous transformation were determined by three radiologists in blinded and independent fashion in 30 patients. Interobserver agreement was measured by the kappa index and intraclass correlation coefficient. Interobserver agreement was considered substantial $(\kappa=0.714-0.795)$ for portal vein thrombosis and perfect $(\kappa=1)$ for cavernous transformation. Interobserver agreement measured by the intraclass correlation coefficient was excellent for longitudinal diameter of the spleen $(r=0.828-0.869)$ and splenic index $(r=0.816-0.905)$ and varied from fair to almost perfect for diameter of the portal $(r=0.622-0.675)$, splenic $(r=0.573-0.913)$ and superior mesenteric ( $r=0.525-0.607)$ veins. According to the results, ultrasound is a highly reproducible method for the main morphological parameters of portal hypertension in schistosomiasis patients.

Key words: schistosomiasis - ultrasound - portal hypertension - spleen

According to the World Health Organization (WHO), schistosomiasis affects more than 200 million people worldwide, approximately $10 \%$ of whom present with the severe form of the disease (Ross et al. 2002). In Brazil, data from the Schistosomiasis Control Program of the Ministry of Health showed a prevalence of $5.4 \%$ in 2002 (Coura \& Amaral 2004), exclusively of the mansonic subtype.

During the life cycle of Schistosoma mansoni, adult worms colonize the terminal venules of the intestines and lay their eggs, which reach the liver through the portal circulation. The result is an intense periportal inflammatory reaction responsible for the development of the essential pathological lesion of schistosomiasis: intra and extra-hepatic periportal fibrosis, leading to presinusoidal blockage and high resistance to portal blood flow (Patel et al. 1993, Martins et al. 2000, Ross et al. 2002, Machado et al. 2004, Bezerra et al. 2007).

Chronic schistosomiasis infection can present in two ways: a less aggressive hepatointestinal form and a more severe hepatosplenic form that presents as portal hypertension, portosystemic collateral circulation, ascitis, upper digestive bleeding and splenomegaly (Pereira et al. 1998). Elevated resistance to portal blood flow in schistosomiasis is a cause of portal vein thrombosis and can cause collateral circulation in the portal hilum known as

+ Corresponding author: giuseppe dr@uol.com.br

Received 13 October 2008

Accepted 9 December 2009 cavernous transformation (Senzolo et al. 2006). Upper digestive bleeding from portal hypertension is the main cause of death from this disease and not rarely is the first clinical manifestation (Tsubaki et al. 2007).

Abdominal ultrasound is important in the evaluation of portal hypertension in patients with chronic hepatic schistosomiasis, in addition to showing abnormalities of the liver, spleen and gallbladder that are consistent with its diagnosis (Pereira et al. 1998). Ultrasound is considered a fast, safe and non-invasive technique for evaluating these parameters and is important in the diagnosis and treatment of hepatosplenic schistosomiasis (Lambertucci et al. 2000).

Some ultrasound parameters, such as spleen size, diameters of the splenic and portal veins and presence of portosystemic collateral circulation, are related to the quantification of the risk of bleeding from esophageal varices in patients with hepatosplenic schistosomiasis (Richter et al. 1992, Abdel-Wahab et al. 1993, Domingues et al. 1993, Eltoum et al. 1994, Richter et al. 1998, Martins et al. 2000). Prophylactic treatment of portal hypertension is particularly important when the costs and inconveniences of drug treatment and complications from endoscopic and surgical procedures are considered (Martins et al. 2000).

Although many studies have evaluated the importance of ultrasound-derived values for portal vein and splenic vein caliber and spleen dimensions, no studies, to our knowledge, have precisely evaluated the method's reproducibility. Studies evaluating reproducibility are important to identify diagnostic tests that may require improvements in technique. Reproducibility of a method, measured by interobserver and intraobserver agreement, indicates the method's precision (but not its accuracy). 
Establishing the precision of a diagnostic method is crucial when it is intended for use in clinical practice, such as ultrasound in the evaluation of schistosomiasis patients.

The purpose of this study was to assess the reproducibility of ultrasound in the evaluation of the main morphological parameters of portal hypertension, including spleen size and the diameters of the portal, splenic and superior mesenteric veins, in patients with hepatosplenic schistosomiasis.

\section{PATIENTS, MATERIALS AND METHODS}

Between May-July 2007, we performed a prospective, cross-sectional and observational study evaluating 30 patients with hepatosplenic schistosomiasis. The sample included 15 men and 15 women (mean age 45 years; range, 25-68 years). Inclusion criteria were age $\geq 18$ years and diagnosis of schistosomiasis by rectal biopsy or positive stool test. All patients had a strong epidemiological history, defined as coming from schistosomiasis endemic areas. Exclusion criteria were history of alcohol consumption (more than $160 \mathrm{~g}$ per week), positive serology for hepatitis virus $\mathrm{B}$ or $\mathrm{C}$, autoimmune hepatitis and use of hepatotoxic drugs, in order to exclude any other source of underlying liver disease. In addition, patients with a previous history of splenectomy or portosystemic shunt surgery were not included. The study was approved by the Institutional Review Board for Human Research and all patients provided written informed consent.

Ultrasound technique - In all patients, conventional B-mode and color Doppler ultrasound was performed using an Envisor platform (Philips Medical System, Best, The Netherlands) with a 3.5-5 MHz multifrequency convex transducer. We adopted the imaging planes standardized by the WHO for ultrasound evaluation of the spleen and splanchnic vascular system in schistosomotic portal hypertension. Patients had fasted for at least $4 \mathrm{~h}$ before the examination. The internal diameter (inner wall to inner wall) of the portal vein was measured with the patient supine, with subcostal and intercostal oblique views of the main portal branch halfway to its bifurcation, during the same respiratory phase (Fig. 1). We also measured the caliber of the splenic vein at the level of the pancreatic body and the superior mesenteric vein immediately before the splenomesenteric junction. To evaluate the presence or absence of portal vein thrombosis and collateral circulation on the portal bed (cavernous transformation), we used color Doppler, with an angle of insonation varying between $45-60^{\circ}$, where measurement of the portal vein was performed (Fig. 2).

All measurements of the splenoportal vessel diameters were performed with the patient in basal condition (quiet breathing, supine position and fasting) to minimize factors that could interfere with this measurement (Machado et al. 2004). We considered the upper limits of normality to be $12 \mathrm{~mm}$ for the portal vein and $9 \mathrm{~mm}$ for the splenic and superior mesenteric veins (Pinto-Silva et al. 1994).

Size of the spleen was measured with the patient supine, with left subcostal views, on the mid-axillary and axial hemiclavicular line on the longitudinal and transverse planes, respectively. All measurements were performed on cross-sections through the splenic hilum with the intention of creating a constant reference point for repetitive measurements (Fig. 3). Measurements performed with the cross-sections, although not standardized, were used in the study because of the greatly enlarged dimensions of the spleen in these patients. From these unidimensional measurements, we calculated the splenic index, which is the product of the longitudinal, transverse and anteroposterior diameters of the spleen (the upper limit of normality is 480) (Lackner et al. 1980, Bezerra et al. 2005).

Standardization of test analysis - The tests were performed in random order and by two independent examiners, blinded to the patients' disease. The two examiners had at least three years of experience in abdominal ultrasound (obs1 and obs2), previously trained in the test's analytical method. A new test was performed by a radiologist with eight years of experience (obs3), with prior knowledge of the patients' disease. Maximum time between tests was $60 \mathrm{~min}$.

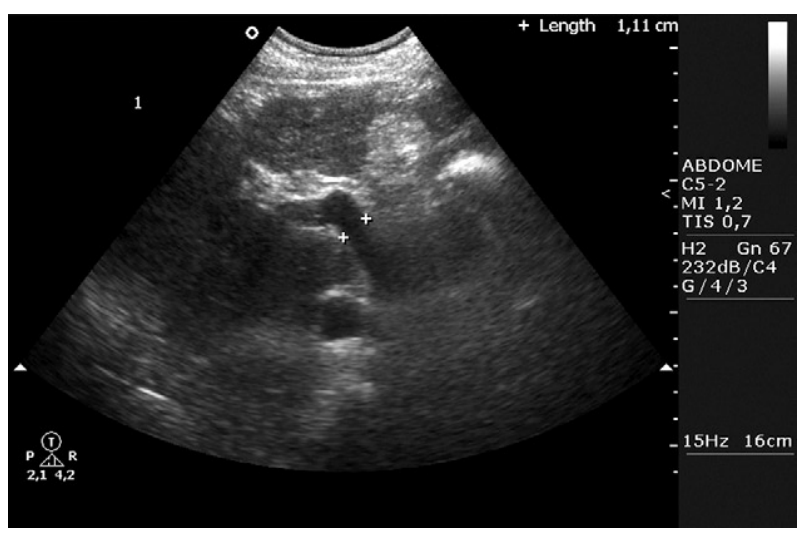

Fig. 1: oblique cut of the hepatic hilum to measure the caliber of the portal vein.

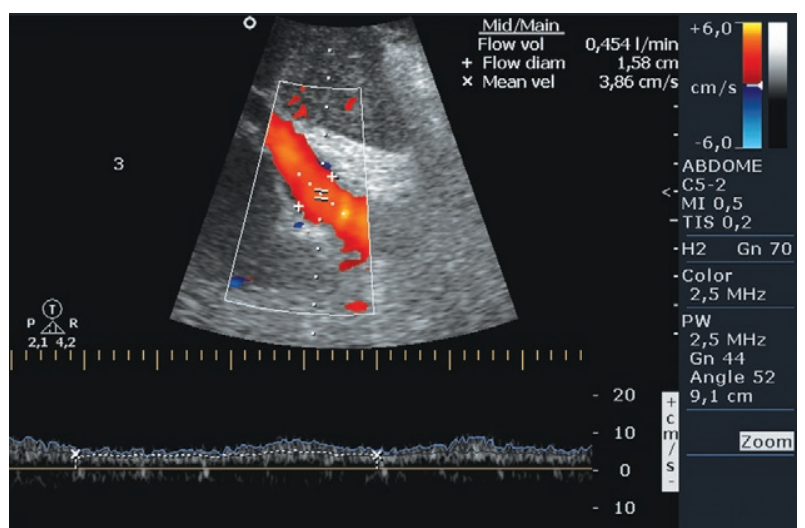

Fig. 2: oblique cut with Doppler ultrasound for the evaluation of portal thrombosis. Portal flow was preserved. 


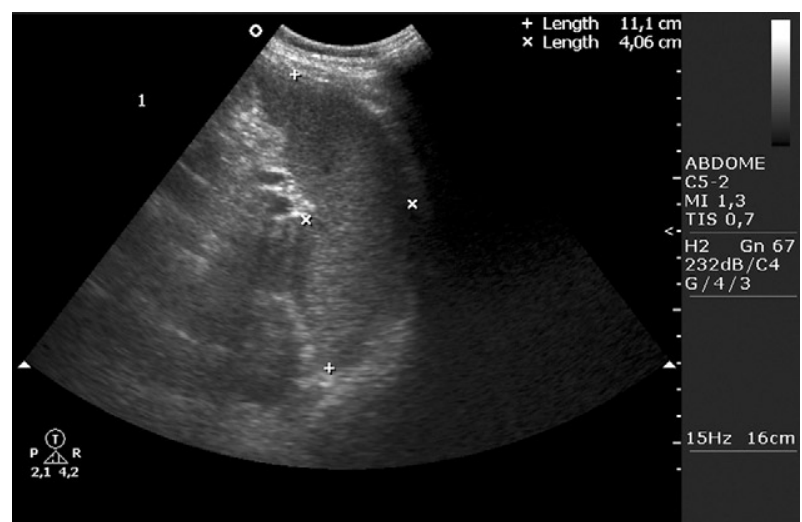

Fig. 3: transversal cut at the splenic hilum to measure the transversal and anteroposterior diameters of the spleen and to allow, together with the measurement of the longitudinal diameter, the calculation of the splenic index.

Statistical analysis - Statistical analysis for measurement of interobserver agreement for the qualitative variables (portal vein thrombosis and cavernous transformation) was performed using kappa statistics $(\kappa)$. The kappa index presents values between $0-1$ and was interpreted as poor $(\kappa=0)$, slight $(0.01-0.2)$, fair $(0.21-0.4)$, moderate (0.41-0.6), substantial (0.61-0.8), almost perfect (0.81-1) and perfect (1) (Landis \& Koch 1977).

Calculation of the kappa index required comparison of data obtained by grouping the examiners into pairs (obs $1 \times$ obs 2 ; obs $1 \times$ obs 3 ; obs $2 \times$ obs 3 ). From these data, we obtained the lowest and highest kappa values for each qualitative variable.

Statistical analysis of interobserver agreement for quantitative variables (longitudinal diameter of the spleen, splenic index, portal vein diameter, splenic vein diameter and superior mesenteric vein diameter) was performed using the intraclass correlation coefficient and respective $95 \%$ confidence interval $(95 \% \mathrm{CI})$. Results were interpreted as poor $(\mathrm{r}<0.4)$, regular $(0.41$ 0.75 ) or excellent (0.76-1) (Shrout \& Fleiss 1979).

The intraclass correlation coefficient was calculated two ways. First, the values were compared by grouping the examiners in pairs, as with the kappa statistics. Second, values obtained by the three examiners were compared at the same time, for each variable.

\section{RESULTS}

Interobserver agreement was considered substantial $(\kappa=0.714-0.795)$ for presence or absence of portal vein thrombosis and perfect $(\kappa=1)$ for cavernous transformation (Table I). The three examiners observed cavernous transformation in 46\% (6/13) of patients with portal vein thrombosis.

Interobserver agreement for longitudinal diameter of the spleen and splenic index was almost perfect, as shown in Tables II and III.

Of the 30 schistosomiasis patients analyzed, portal vein diameter was not measured in seven patients because of complete thrombosis. Thus, measurements were
TABLE I

Interobserver agreement for qualitative variables (kappa index) calculated from the comparison of data by pairing observers

obs $1 \times$ obs 2 obs $1 \times$ obs 3 obs $2 \times$ obs 3

\begin{tabular}{lccc}
\hline $\begin{array}{l}\text { Portal vein thrombosis } \\
\text { Cavernous }\end{array}$ & 0.791 & 0.795 & 0.714 \\
transformation & 1 & 1 & 1 \\
\hline
\end{tabular}

performed in 23 patients. In six of the 13 patients with partial portal thrombosis, it was possible to measure the portal vein diameter (Table IV).

During evaluation of the superior mesenteric vein, it was not possible to measure the diameter in two patients because of excessive patient fat and gas interposition.

Interobserver agreement measured by the intraclass correlation coefficient showed results varying from regular to excellent for the diameters of the portal, splenic and superior mesenteric veins (Tables II, III).

\section{DISCUSSION}

Various studies have focused on ultrasound in an effort to reveal abnormalities in the abdominal organs consistent with the diagnosis of chronic schistosomiasis and to identify parameters that relate to quantification of the risk of digestive bleeding (Richter et al. 1992, 1998, Abdel-Wahab et al. 1993, Domingues et al. 1993, Eltoum et al. 1994, Martins et al. 2000). Although some studies show the importance of this diagnostic method, especially regarding evaluation of portal hypertension, its diagnostic precision has not been closely assessed. In a recent literature review that included studies of interobserver and intraobserver agreement in hepatology, the authors concluded that the reproducibility of ultrasound outcomes was not sufficiently studied, with only one article addressing ultrasound reproducibility in the evaluation of portal hypertension (Tissot et al. 1995, Winkfield et al. 2003).

We performed a new literature review in which we identified only four articles that analyzed interobserver agreement for portal vein diameter, splenic vein diameter and/or size of the spleen in patients with chronic liver disease. Of these, only one was related to evaluation of morphological parameters for portal hypertension. The other studies focused on measuring Doppler reproducibility and marginally evaluating portal vein diameter and size of the spleen (Fernández et al. 1991, Sabbà et al. 1995, Tissot et al. 1995, O'Donohue et al. 2004). It should be noted that the results obtained in these studies were poor, perhaps because they were performed more than a decade ago and thus involved equipment with lower resolution than available at present.

The current paper showed high interobserver agreement for the target parameters (portal vein diameter, splenic vein diameter, spleen size and portal vein thrombosis), especially regarding interobserver agreement for the evaluation of cavernous transformation, spleen size and splenic index, which was excellent or almost perfect. 


\section{TABLE II}

Interobserver agreement for quantitative variables (intraclass correlation coefficient calculated from the comparison of data between paired observers)

\begin{tabular}{lccc}
\hline & obs1 $\times$ obs2 & obs1 $\times$ obs3 & obs2 $\times$ obs3 \\
\hline Longitudinal diameter of the spleen & 0.869 & 0.866 & 0.828 \\
Splenic index & 0.816 & 0.905 & 0.840 \\
Portal vein diameter & 0.675 & 0.654 & 0.622 \\
Splenic vein diameter & 0.573 & 0.913 & 0.592 \\
Superior mesenteric vein diameter & 0.599 & 0.710 & 0.525
\end{tabular}

\section{TABLE III}

Interobserver agreement for quantitative variables calculated from the comparison of data from all three observers

\begin{tabular}{lc}
\hline Variables & $\begin{array}{c}\text { Intraclass correlation } \\
\text { coefficient } \\
\text { obs1 } \times \text { obs } 2 \times \text { obs3 }\end{array}$ \\
\hline Longitudinal diameter of the spleen & 0.854 \\
Splenic index & 0.853 \\
Portal vein diameter & 0.651 \\
Splenic vein diameter & 0.694 \\
Superior mesenteric vein diameter & 0.607
\end{tabular}

the minimum, maximum and mean (among the three examiners) measurements obtained for each quantitative variable are described in Table IV.

\section{TABLE IV}

The minimum, maximum and mean measurement obtained for each quantitative variable

\begin{tabular}{lccc}
\hline Variables & Minimum & Maximum & Mean \\
\hline $\begin{array}{l}\text { Longitudinal diameter } \\
\text { of the spleen }(\mathrm{cm})\end{array}$ & 9.4 & 30 & 18.8 \\
Splenic index & 373.6 & 6493.0 & 1797.8 \\
Portal vein diameter $(\mathrm{cm})$ & 0.6 & 2 & 1.36 \\
$\begin{array}{l}\text { Splenic vein diameter }(\mathrm{cm}) \\
\text { Superior mesenteric }\end{array}$ & 0.5 & 1.9 & 1.17 \\
vein diameter $(\mathrm{cm})$ & 0.5 & 2.5 & 1.26 \\
\hline
\end{tabular}

The importance of B-mode ultrasound is currently acknowledged for the evaluation of ultrasound parameters related to the risk of upper digestive tract bleeding. Our results, in combination with the relatively low cost and wide availability of ultrasound, further emphasize its role in the evaluation of patients with schistosomiasis.

Our study has two main limitations. One was the relatively small number of patients and the other was the previous training of the less experienced observers, which may have increased the interobserver agreement.
In conclusion, based on these results, we can state that abdominal ultrasound is a highly reproducible method for analyzing the main morphological parameters of portal hypertension and can be used reliably in the evaluation of patients with schistosomiasis.

\section{REFERENCES}

Abdel-Wahab MF, Esmat G, Farrag A, el-Boraey Y, Strickland GT 1993. Ultrasonographic prediction of esophageal varices in schistosomiasis mansoni. Am J Gastroenterol 88: 560-563.

Bezerra AS, D'Ippolito G, Caldana RP, Cecin AO, Ahmed M, Szejnfeld J 2007. Chronic hepatosplenic schistosomiasis mansoni: magnetic resonance imaging and magnetic resonance angiography findings. Acta Radiol 48: 125-134.

Bezerra AS, D’Ippolito G, Faintuch S, Szejnfeld J, Ahmed M 2005. Determination of splenomegaly by CT: is there a place for a single measurement? Am J Roentgenol 184: 1510-1513.

Coura JR, Amaral RS 2004. Epidemiological and control aspects of schistosomiasis in Brazilian endemic areas. Mem Inst Oswaldo Cruz 99 (Suppl. I): 13-19.

Domingues AL, Lima AR, Dias HS, Leao GC, Coutinho A 1993. An ultrasonographic study of liver fibrosis in patients infected with Schistosoma mansoni in North-east Brazil. Trans R Soc Trop Med Hyg 87: 555-558.

Eltoum IA, Taha TE, Saad AM, Suliman SM, Bennett JL, Nash TE, Homeida MM 1994. Predictors of upper gastrointestinal bleeding in patients with schistosomal periportal fibrosis. Br J Surg 81: 996-999.

Fernández M, Chesta J, Jirón MI, Mánquez P, Brahm J 1991. Liver cirrhosis and portal hypertension: non-invasive measurement of blood flow in the portal vein with Doppler-duplex. Rev Med Chil 119: 524-529.

Lackner K, Brecht G, Janson R, Scherholz K, Lützeler A, Thurn P 1980. The value of computer tomography in the staging of primary lymph node neoplasms. Rofo 132: 21-30.

Lambertucci JR, Serufo JC, Gerspacher-Lara R, Rayes AA, Teixeira R, Nobre V, Antunes CM 2000. Schistosoma mansoni: assessment of morbidity before and after control. Acta Trop 77: 101-109.

Landis JR, Koch GG 1977. The measurement of observer agreement for categorical data. Biometrics 33: 159-174.

Machado MM, Rosa ACF, Barros N, Azeredo LM, Rosa JBF, Cerri LMO, Chammas MC, Daher MT, Daher RT, Saad WA, Cerri GG 2004. Doppler evaluation in portal hypertension. Radiol Bras 37: 35-39.

Martins RD, Szejnfeld J, Lima FG, Ferrari AP 2000. Endoscopic, ultrasonographic and US-Doppler parameters as indicators of 
variceal bleeding in patients with schistosomiasis. Dig Dis Sci 45: 1013-1018

O’Donohue J, Ng C, Catnach S, Farrant P, Williams R 2004. Diagnostic value of Doppler assessment of the hepatic and portal vessels and ultrasound of the spleen in liver disease. Eur J Gastroenterol Hepatol 16: 147-155.

Patel SA, Castillo DF, Hibbeln JF, Watkins JL 1993. Magnetic resonance imaging appearance of hepatic schistosomiasis, with ultrasound and computed tomography correlation. Am J Gastroenterol 88: 113-116.

Pereira LM, Domingues AL, Spinelli V, McFarlane IG 1998. Ultrasonography of the liver and spleen in Brazilian patients with hepatosplenic schistosomiasis and cirrhosis. Trans $R$ Soc Trop Med Hyg 92: 639-642.

Pinto-Silva RA, Abrantes WL, Antunes CM, Lambertucci JR 1994. Sonographic features of portal hypertension in schistosomiasis mansoni. Rev Inst Med Trop Sao Paulo 36: 355-361.

Richter J, Correia Dacal AR, Vergetti Siqueira JG, Poggensee G, Mannsmann U, Deelder A, Feldmeier H 1998. Sonographic prediction of variceal bleeding in patients with liver fibrosis due to Schistosoma mansoni. Trop Med Int Health 3: 728-735.

Richter J, Zwingenberger K, Ali QM, Lima W de M, Dacal AR, de Siqueira GV, Doehring-Schwerdtfeger E, Feldmeier H 1992. Hepatosplenic schistosomiasis: comparison of sonographic findings in Brazilian and Sudanese patients - correlation of sonographic findings with clinical symptoms. Radiology 184: 711-716.

Ross AG, Bartley PB, Sleigh AC, Olds GR, Li Y, Williams GM, McManus DP 2002. Schistosomiasis. N Engl J Med 346: 1212-1220.

Sabbà C, Merkel C, Zoli M, Ferraioli G, Gaiani S, Sacerdoti D, Bolondi L 1995. Interobserver and interequipment variability of echoDoppler examination of the portal vein: effect of a cooperative training program. Hepatology 21: 428-433.

Senzolo M, Tibbals J, Cholongitas E, Triantos CK, Burroughs AK, Patch D 2006. Transjugular intrahepatic portosystemic shunt for portal vein thrombosis with and without cavernous transformation. Aliment Pharmacol Ther 23: 767-775.

Shrout PE, Fleiss JL 1979. Intraclass correlations: uses in assessing rater reliability. Psychol Bull 86: 420-428.

Tissot O, Aubé C, Namour A, Blanc F, Dauver A, Calès P 1995. Interobserver concordance of echographic semiology in liver cirrhosis. Gastroenterol Clin Biol 19: 291-296.

Tsubaki T, Sato S, Fujikawa H, Kawashima Y, Kitazawa E, Takai A, Kikuchi K, Morizane T, Miyakawa H 2007. Values of Doppler sonography predicts high risk variceal bleeding in patients with viral cirrhosis. Hepatogastroenterology 54: 96-99.

Winkfield B, Aubé C, Burtin P, Calès P 2003. Inter-observer and intra-observer variability in hepatology. Eur J Gastroenterol Hepatol 15: 959-966. 